\title{
Supporting Information: Coordination of substrate binding and protonation in the $N$. gonorrhoeae MtrD efflux pump controls functionally rotating transport mechanism
}

Stephen J. Fairweather ${ }^{\mathrm{a}, \mathrm{b} \#}$, Vrinda Gupta ${ }^{\mathrm{a}}$, Mohsen Chitsaz ${ }^{\mathrm{c}}$, Lauren Booth ${ }^{\mathrm{a}}$, Melissa H. Brown ${ }^{\mathrm{c}}$, Megan L. O'Mara*a

${ }^{a}$ Research School of Chemistry, The Australian National University, Canberra, ACT, Australia.

${ }^{b}$ Research School of Biology, The Australian National University, Canberra, ACT, Australia.

${ }^{\mathrm{c}}$ College of Science and Engineering, Flinders University, Bedford Park, SA, Australia.

\# co-first authors

*Corresponding author: megan.omara@anu.edu.au 


\section{Table of Contents}

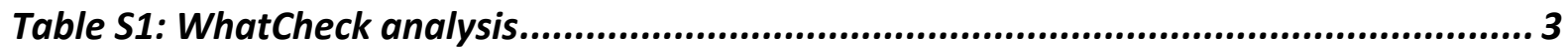

Figure S1: Sequence alignment and conservation histogram of RND efflux pumps............ 4

Figure S2: Protein expression of D405C MtrD mutant............................................... 5

Figure S3: Crystal contacts in the symmetric MtrD structure ........................................6

Figure S4: MtrD structural overview ............................................................. 7

Figure S5: Coarse-grain simulations RMSD analysis ......................................... 8

Figure S6: Time-correlation of proton relay network and extrusion gate distances ........... 9

Figure S7: Time-correlation of proton relay network, extrusion gate and binding cleft distances ................................................................................................ 10

Figure S8: TMD rotation in deprotonated monomers ............................................ 11

Figure S9: Time-correlation of TMD rotation and inter-monomeric distances................... 12 


\section{Supplementary Figures}

Table S1: WhatCheck analysis. MtrD protein quality validation (WhatCheck) for pre- and postsimulation structures

\begin{tabular}{|c|c|c|c|c|c|}
\hline \multirow{2}{*}{$\begin{array}{c}\text { MtrD } \\
\text { Structure or } \\
\text { Simulation }\end{array}$} & \multicolumn{5}{|c|}{ Protein Quality Parameter (WhatCheck) } \\
\hline & $\begin{array}{c}1^{\text {st }} \text { gen } \\
\text { packing }\end{array}$ & $\begin{array}{l}2^{\text {nd }} \text { gen } \\
\text { packing }\end{array}$ & $\begin{array}{c}\text { Ramachan- } \\
\text { dran }\end{array}$ & $\mathbf{X}_{1} / \mathbf{X}_{2}$ & Backbone \\
\hline $\begin{array}{l}\text { PDB ID: } \\
\text { 4MT1 } \\
\text { symmetric } \\
\text { crystal } \\
\text { structure }\end{array}$ & -1.509 & -1.737 & -4.925 & -6.687 & -2.106 \\
\hline $\begin{array}{l}\text { Back-mapped } \\
\text { MtrD 4MT1 } \\
\text { post CG MD }\end{array}$ & -2.378 & -3.562 & -5.724 & -8.63 & -5.101 \\
\hline System 1 & -1.973 & -2.474 & -5.458 & -7.198 & -2.762 \\
\hline System 2 & -1.935 & -2.360 & -5.705 & -6.976 & -3.143 \\
\hline System 3 & -1.888 & -2.338 & -5.414 & -7.052 & -3.075 \\
\hline System 4 & -1.836 & -2.321 & -5.433 & -6.904 & -2.598 \\
\hline System 5 & -1.903 & -2.325 & -5.238 & -6.986 & -2.586 \\
\hline $\begin{array}{l}\text { PDB ID: } \\
\text { 6VKT } \\
\text { asymmetric } \\
\text { cryo-EM } \\
\text { structure }\end{array}$ & -1.414 & -2.128 & -4.542 & -6.000 & -1.187 \\
\hline $\begin{array}{c}\text { PDB ID: } \\
\text { 6VKS } \\
\text { MtrD } \\
\text { asymmetric } \\
\text { cryo-EM } \\
\text { structure }\end{array}$ & -0.359 & -0.822 & -1.885 & -2.878 & -0.944 \\
\hline
\end{tabular}




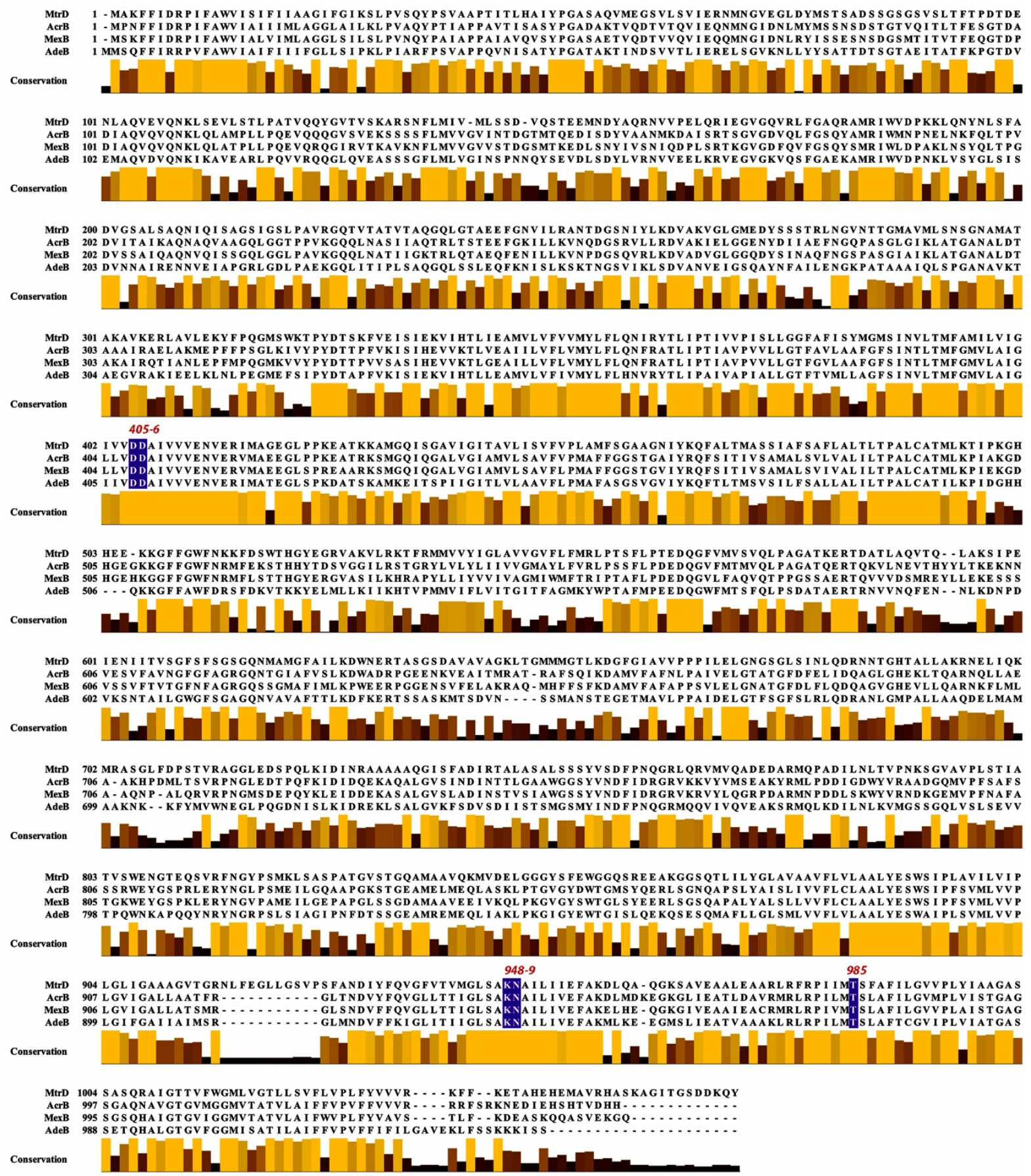

Figure S1: Sequence alignment and conservation histogram of RND efflux pumps. The sequence alignment of four RND efflux pumps with solved structural coordinates, namely MtrD (PDB ID: 4MT1, 6VKS, 6VKT), AcrB (>90 structures deposited in the Protein Data Bank under different conditions, with and without associated proteins), MexB (10 structures deposited), AdeB (7 structures deposited). The height of the histogram below each row of the sequence alignment 
correspond to the sequence conservation per residue. Residues involved in the proton relay network are highlighted in purple. The proton relay switch numbering corresponds to the position in MtrD, and the proton relay switch residues display $100 \%$ sequence identity across the RND homologues shown here.

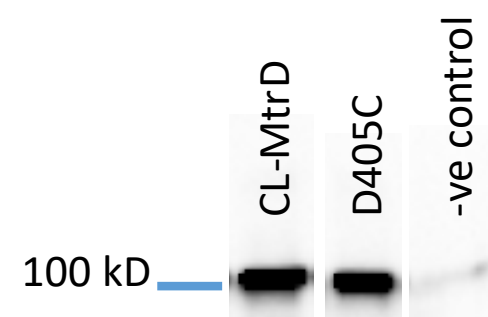

Figure S2: Protein expression of D405C MtrD mutant. Western blot analysis demonstrating

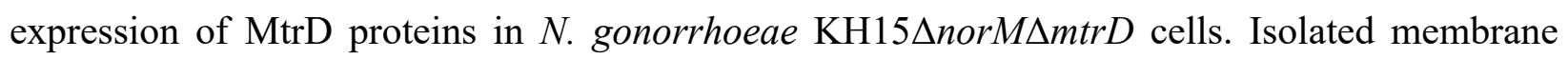
fractions were separated by SDS-PAGE and the blot was probed with an anti-6xHis epitope tag (rabbit) antibody using standard methods. ${ }^{1}$ The full-length $\mathrm{MtrD}$ protein has a predicted molecular mass of nearly $114 \mathrm{kDa} .{ }^{2}$ Expression of MtrD was determined by presence of a protein band at position approximately $100 \mathrm{kDa}$ using protein molecular mass standard (Precision Plus Protein Dual Colour; Bio-Rad), indicated on the left in kDa. CL-MtrD represents expression of the cysteine-less $\mathrm{MtrD}_{6 \mathrm{His}}$ protein (referred to as wild-type). Expression of D405C mutant is shown in the second lane, and -ve control are KH15 nnorM $\Delta m t r D$ cells with no MtrD expression. 

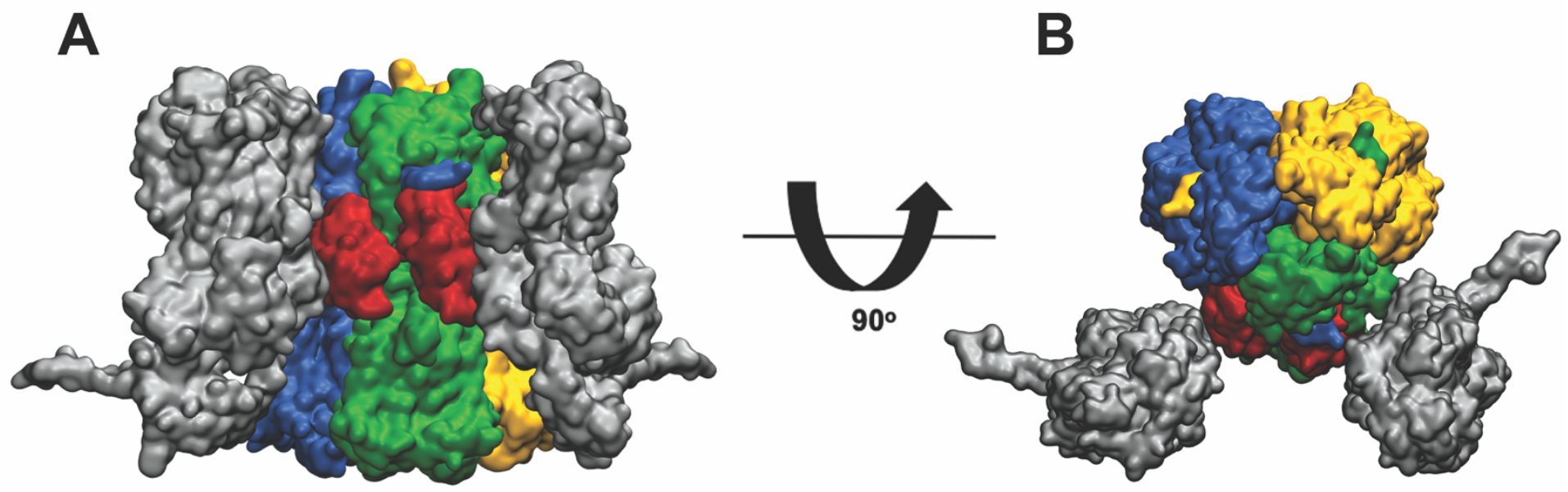

Figure S3: Crystal contacts in the symmetric MtrD structure. Crystal contacts between adjacent proteins from the rebuilt unit cell of the symmetric MtrD structure (PDB ID: 4MT1), viewed from the (A) sideview and (B) top-down view. The three monomers of the central MtrD are coloured green, yellow and blue and the PC1 and PC2 subdomains are shown in red. Crystallographic contacts form between the Monomers from adjacent MtrD proteins (grey surface) in the crystal lattice form contacts with the PC1 and PC2 domains of adjacent MtrD trimers. 

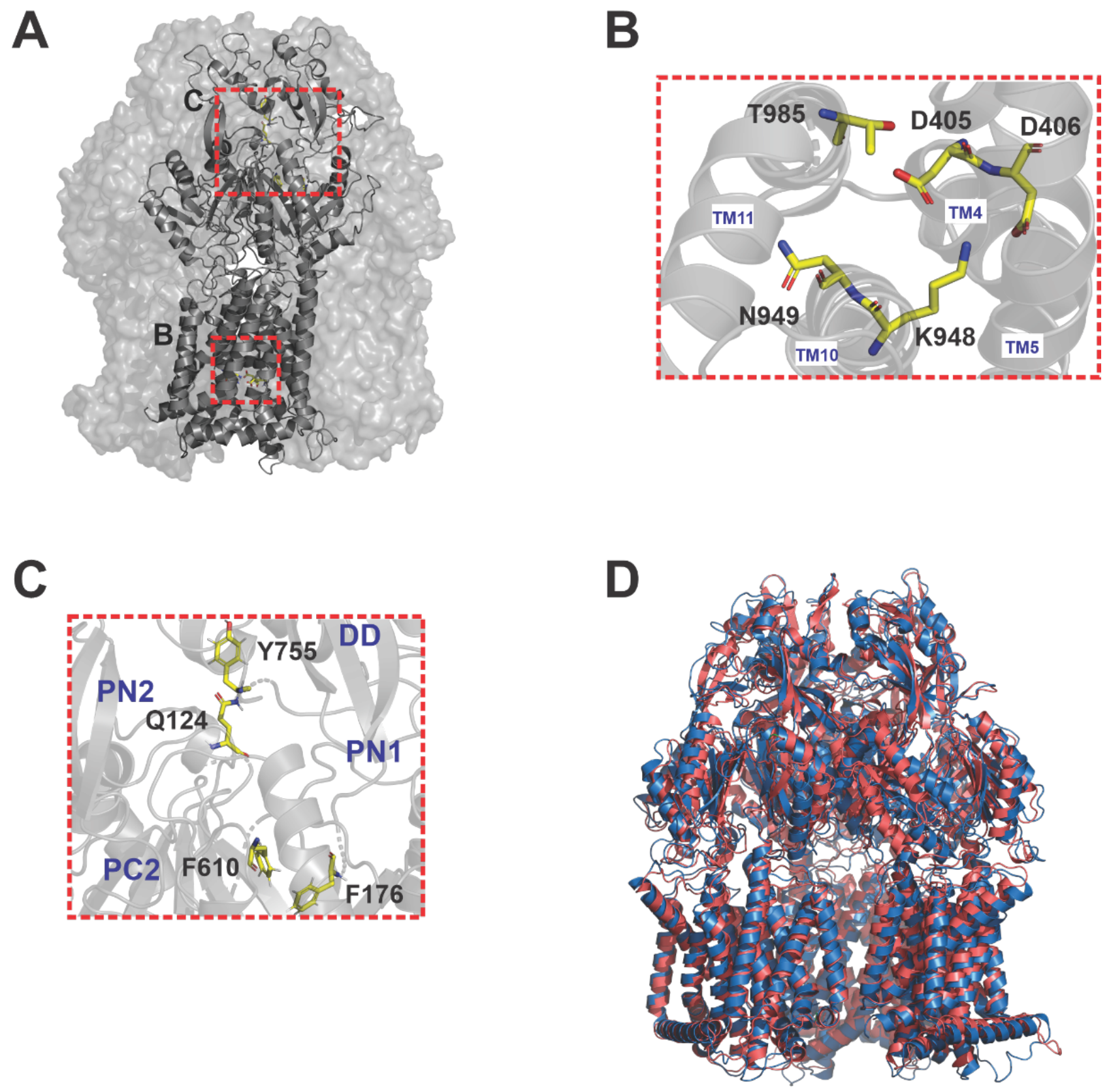

Figure S4: MtrD structural overview. (A) The functional regions of MtrD involved in the conformational transitions needed for the functionally rotating transport mechanism. (B) Close-up view of the proton relay network (D405, D406, K948, K949 and T985) and (C) binding cleft (F176-F610) and extrusion gate (Q124-Y755) residues used in analysis. (D) Alignment between the MtrD symmetric hybrid structure (PDB ID: 4MT1) in pink and asymmetric structure (PDB ID: 6VKS) in blue along the backbone heavy atoms. 


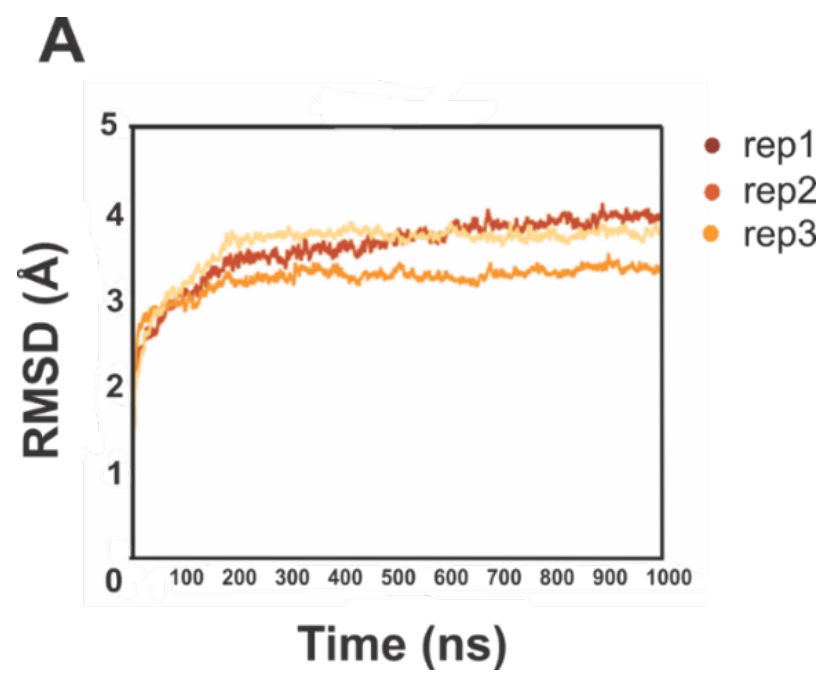

Figure S5: Coarse-grain simulations RMSD analysis. The protein backbone RMSD for the symmetric MtrD trimer (PDB ID: 4MT1) across the $1 \mu$ s of GoMARTINI coarse-grained simulations. RMSD values are shown individually for each of the three system replicates. The GoMARTINI forcefield replaces the elastic models of restricted movement with a more physically motivated model that allows for extensive thermal motions and better accuracy of protein dynamics. 
A
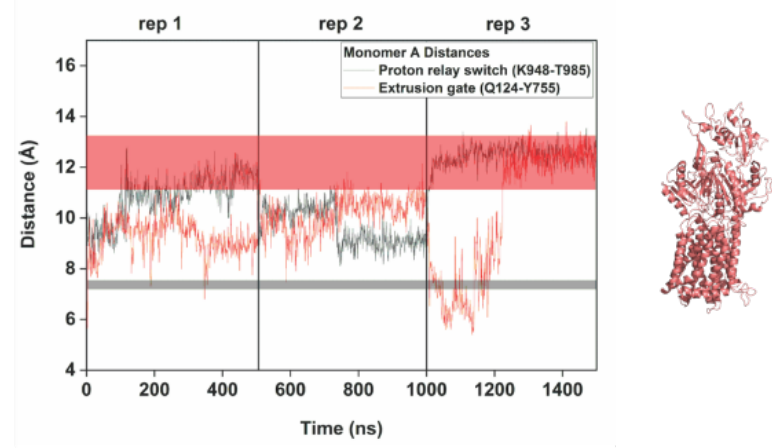

Extrusion distances

K948-T985 (disengaged)

Q124-F755 (open)

\section{B}
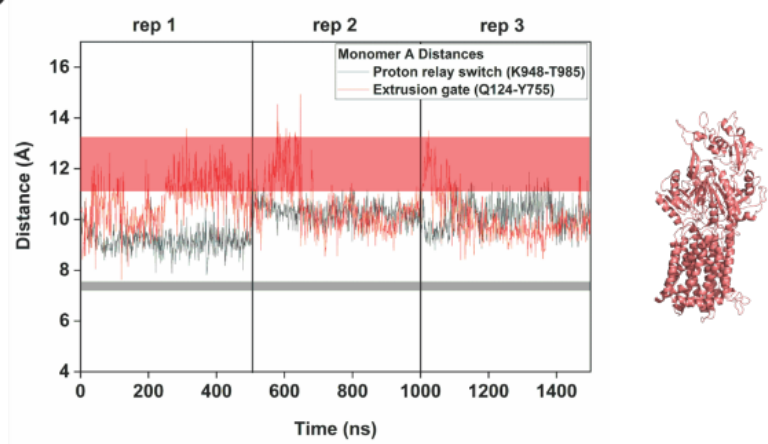

Extrusion distances

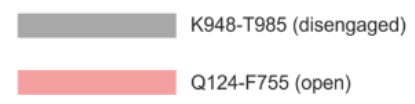

Figure S6: Time-correlation of proton relay network and extrusion gate distances. Coupling between the monomer A proton relay network (grey) and extrusion gate (red) distances across the concatenated trajectory of three $500 \mathrm{~ns}$ system replicates. (A) Simulation System 2, monomer A and (B) simulation System 3, monomer A. 
A

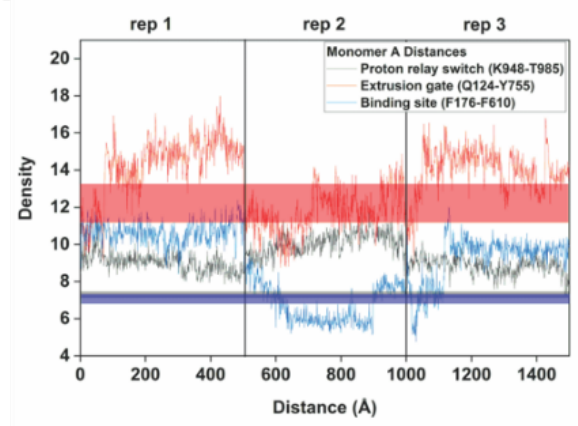

B

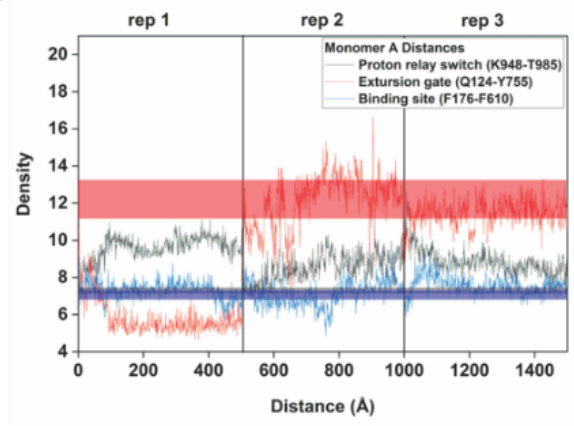

C
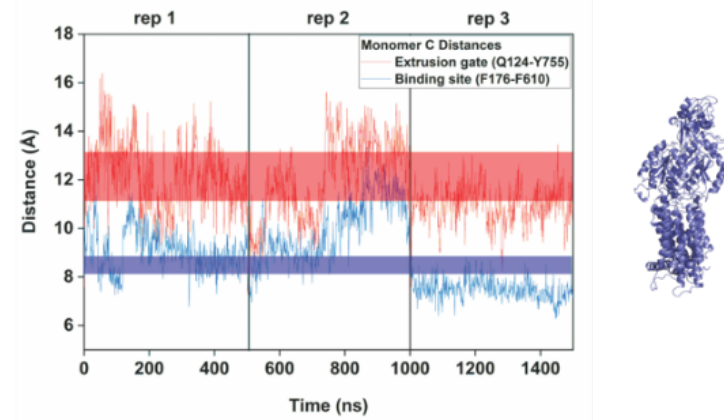

Extrusion distances

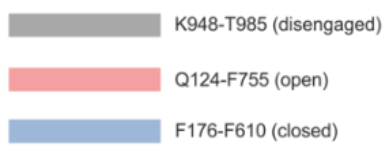

Figure S7: Time-correlation of proton relay network, extrusion gate and binding cleft distances. Coupling between the monomer A and monomer C proton relay network (grey), extrusion gate (red) and binding cleft (blue) distances across the concatenated trajectory of three 500 ns system replicates. (A) Simulation System 4, monomer A, (B) simulation System 5, monomer A and (C) simulation System 5, monomer C. 

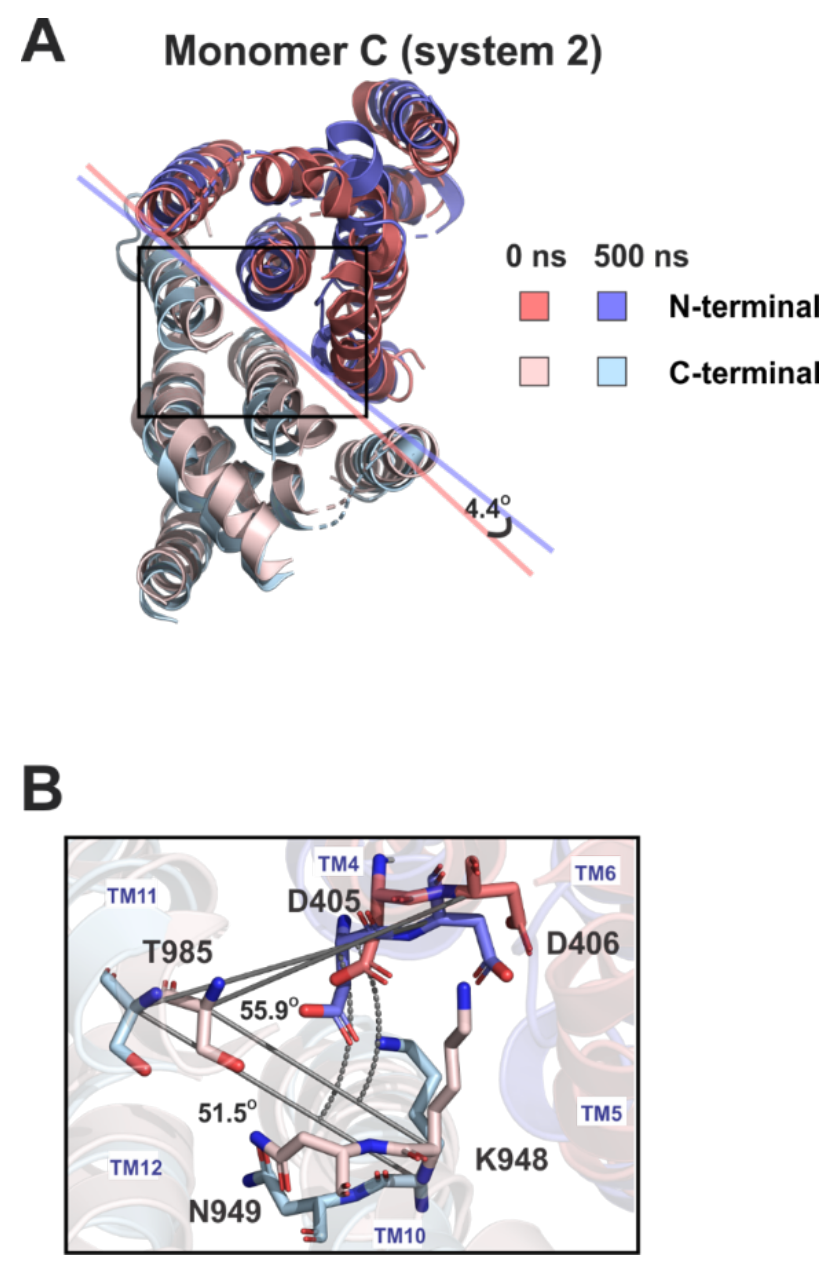

Figure S8: TMD rotation in deprotonated monomers. Deprotonated monomer C of System 2 showed little TM domain helical rotation. The MtrD TM domains forming the proton relay network are viewed from the cytosolic side of the transporter. (A) The $\Delta$ D406-T985-K948 $\mathrm{C}_{\alpha}$ angle shows a $4.4^{\circ}$ rotation about the pseudosymmetrical axis of the TM domains between the initial (red) and final (blue) frames. (B) Overlay of the initial (red) and final (blue) frames of the proton relay network residues in monomer $\mathrm{C}$ of System 2 indicate a change in the $\Delta \mathrm{D} 406-\mathrm{T} 985-$ $\mathrm{K} 948 \mathrm{C}_{\alpha}$ angle from $55.9^{\circ}$ to $51.5^{\circ}$. 

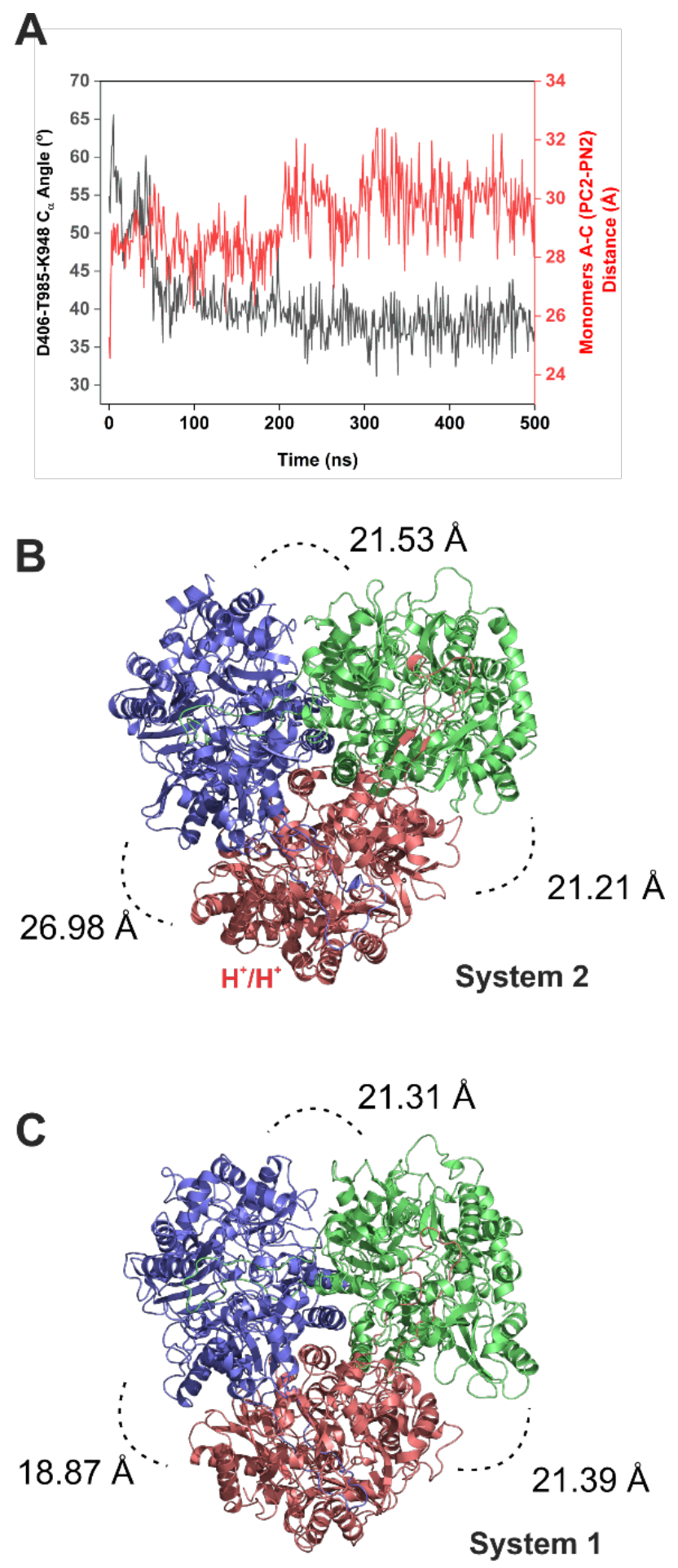

Figure S9: Time-correlation of TMD rotation and inter-monomeric distances. Protonation and TM domain helical rotation is correlated with an increase in inter-monomeric distance between the binding (blue, monomer C) and extrusion (pink, monomer A) monomers. (A) Coupling between the D406-T985-K948 Ca angle and the inter-monomer distance between monomer A and 
$\mathrm{C}$ in System 2, replicate 2. Average inter-monomeric distances (Table 5) between the access (green, monomer B), binding (blue, monomer C) and extrusion (pink, monomer A) monomers of MtrD in (B) System 2 and (C) System 1.

\section{REFERENCES}

1. Chitsaz, M.; Booth, L.; Blyth, M. T.; O'Mara, M. L.; Brown, M. H., Multidrug resistance in Neisseria gonorrhoeae: Identification of functionally important residues in the MtrD efflux protein. $m$ Bio 2019, 10 (6). DOI: 10.1128/mBio.02277-19.

2. Hagman, K. E.; Lucas, C. E.; Balthazar, J. T.; Snyder, L.; Nilles, M.; Judd, R. C.; Shafer, W. M., The MtrD protein of Neisseria gonorrhoeae is a member of the resistance/nodulation/division protein family constituting part of an efflux system. Microbiology 1997, 143, 2117-25. 